\section{Time-domain terahertz spectroscopy based on asynchronous optical sampling with femtosecond semiconductor disk laser}

\section{R. Gebs, P. Klopp, G. Klatt, T. Dekorsy, U. Griebner and A. Bartels}

A terahertz time-domain spectroscopy (THz-TDS) system using an optically pumped semiconductor disk laser and high-speed asynchronous optical sampling is reported. The combination of these techniques holds considerable promise towards a compact and low-cost implementation of THz-TDS. The spectrometer offers a $333 \mathrm{ps}$ time delay window scanned at $10 \mathrm{kHz}$ and delivers more than $1.2 \mathrm{THz}$ spectral coverage at a data acquisition time of one minute. As a proof-ofprinciple experiment, a $0.8 \mathrm{THz}$ resonance of a frequency-selective surface structure was measured.

Introduction: Terahertz time-domain spectroscopy (THz-TDS) is a wel established technique to determine material properties in the range between $100 \mathrm{GHz}$ and $10 \mathrm{THz}$ with applications in pharmaceutical product inspection [1], label-free readout of DNA sensors [2], gas spectroscopy and sensing [3]. Current systems are based on modelocked bulk or fibre lasers which are relatively expensive and voluminous and may pose a considerable barrier preventing the introduction of $\mathrm{THz}$ TDS into emerging applications outside of scientific laboratories Modelocked semiconductor disk lasers (SDLs, sometimes called vertical extemal cavity surface emitting lasers or VECSELs) are promising candidates for an all-semiconductor alternative source of femtosecond pulses with compact dimensions and low cost $[4,5]$. Such lasers have recently been used in a conventional THz-TDS setup with a mechanical delay stage for time-delay scanning [6]. In addition to the size and cost advantages of femtosecond SDLs, their typically high repetition rates $\left(f_{S D L} \geq 1 \mathrm{GHz}\right)$ are attractive for high-speed asynchronous optical sampling (ASOPS). High-speed ASOPS is a recently developed technique permitting rapid THz-TDS measurements without a mechanical delay stage [7]. A THz-TDS system using high-speed ASOPS has been demonstrated at $2 \mathrm{kHz}$ scan rate with $7 \mathrm{THz}$ spectral coverage and with a signal-to-noise ratio (SNR) of better than $40 \mathrm{~dB}$ in $1 \mathrm{~s}$ of data acquisition time [8]. Two modelocked Ti:sapphire (Ti:Sa) lasers were employed, one as a pump laser to generate pulsed $\mathrm{THz}$ radiation in a photoconductive emitter [9], the other to probe the THz radiation after interaction with a sample in the spectrometer. In a standard highspeed ASOPS system the two lasers have repetition rates $f_{R 1} \simeq f_{R 2} \simeq$ $f_{R}=1 \mathrm{GHz}$ and an actively stabilised repetition rate offset $\Delta f_{R}=f_{R}$ $-f_{R 2}$ of a few kilohertz [7]. The repetition rate offset causes the time delay between pairs of pump and probe pulses to be ramped between zero and $1 / f_{R}$ at the scan frequency $f_{\text {scan }}=\Delta f_{R}$. The time-delay increment from pulse pair to pulse pair is given by $\Delta f_{R} / f_{R}^{2}$ and represents the theoretical lowest time resolution limit. In this Letter, we report a high-speed ASOPS THz-TDS system employing a modelocked SDL, which replaces one Ti:Sa laser and serves as the probe laser, Our experiment is a first step combining the new technologies in an effor towards a high-speed, highly compact and cost-efficient THz-TDS tool.

Experimental setup: Fig, 1 shows the setup of our THz time-domain spectrometer. The Ti:Sa laser is Kerr-lens modelocked and emits $40 \mathrm{fs}$ pulses at a repetition rate of $f_{T i: S a} \simeq 1 \mathrm{GHz}$, has a centre wavelength of $825 \mathrm{~nm}$ and maximum average output power of $900 \mathrm{~mW}$ [7]. The diode-pumped SDL is similar to the lasers presented in $[4,5]$. The $50 \mathrm{~mm}$-long ' $\mathrm{V}$ '-shaped cavity contains an output coupler $10.75 \%$ transmission), an InGaAs/AlGaAs chip as the gain medium, and a semiconductor saturable absorber mirror as the passive modelocker [10]. In the $\mathrm{SDL}, 370$ fs pulses are generated at a repetition rate of $f_{S D L} \approx 3 \mathrm{GHz}$ with a centre wavelength of $1048 \mathrm{~nm}$ and an average output power of $12 \mathrm{~mW}$. To stabilise the repetition rate offset, a small part of both lasers $(\approx 3 \mathrm{~mW})$ is split off and focused on high bandwidth photodiodes (PDI and PD2) to detect the laser repetition rates (see Fig. 1). While the SDL is free-running, the Ti:Sa repetition rate is feedback loop controlled by a piezo-driven mirror. The repetition rate offset lock is performed using the third harmonic of the Ti:Sa repetition rate and the first harmonic of the SDL. The offset locking electronies have been described in [7]. The repetition rate offset is chosen to be $\Delta f_{R}=f_{T i: S_{a}}-f_{S D L} / 3=$ $10 \mathrm{kHz}$, which is also the scan rate. The time-delay increment is given by $\Delta f_{R} / f_{T: S a}^{2}=10 \mathrm{fs}$. THz radiation pulses with about 300 fs duration are generated at $1 \mathrm{GHz}$ repetition rate by illuminating a large-area $\mathrm{THz}$ emitter [9] with approximately $600 \mathrm{~mW}$ of average pump power from the Ti:Sa laser. The $\mathrm{THz}$ radiation is collected and focused by two pairs of parabolic mirrors (MI to M4) onto a (110) ZnTe electrooptic detection crystal. The probe pulse train from the SDL $(9 \mathrm{~mW}$ average power) is used to sample the $\mathrm{THz}$ transient via the Pockels effect in the ZnTe crystal and is detected using a photoreceiver (PD3) with $125 \mathrm{MHz}$ bandwidth. The photoreceiver output is digitised using a $100 \mathrm{MS} / \mathrm{s}$, 14-bit digitiser board $(A / D)$ that is triggered by a $10 \mathrm{kHz}$ TTL level signal from the laser stabilisation electronics [7]. The data acquisition of a single $\mathrm{THz}$ trace requires $1 / \Delta f_{R} \simeq 100 \mu$ s. Several thousands of single traces are averaged to increase SNR. The $3 \mathrm{GHz}$ repetition rate of the probe laser limits the usable time-delay window to $1 / f_{S D L}=333 \mathrm{ps}$. Only one out of three consecutive pulses from the probe laser contributes to the signal, while contributions from the other two pulses are negligible as long as the $\mathrm{THz}$ transient has essentially decayed within the first 333 ps, which is the case in our experiment. As a test, we determine a known transmission resonance of a frequency-selective surface (FSS) structure $[11,12]$, which is inserted in the focused region of the $\mathrm{THz}$ beam.

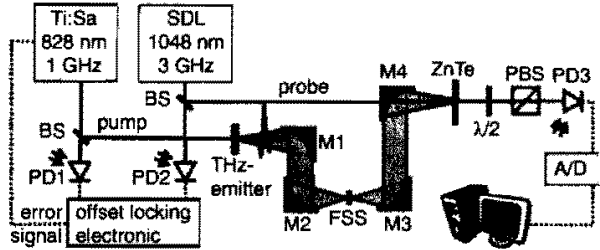

Fig. $1 \mathrm{THz}$ time-domain spectrometer setup including repetition rate offset stabilisation for high-speed ASOPS

Solid lines correspond to optical paths, dashed lines to electronic connections BS: optical beam splitter; PD1, PD2, PD3: amplified photodiodes; $\mathrm{MI}$ to M4: parabolic mirrors; FSS: frequency-selective surface structure; PBS: polarising beam splitter; $\mathrm{A} / \mathrm{D}$ : analogue-to-digital converter

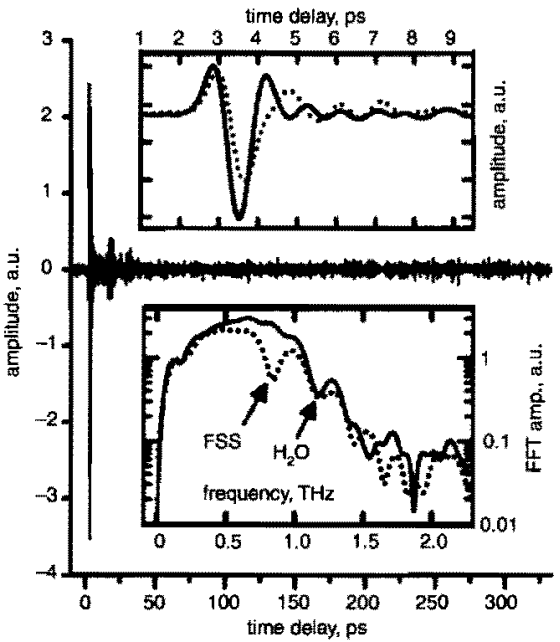

Fig. 2 Detected $\mathrm{THz}$ time-domain transient

Upper inset: Zoom into first $10 \mathrm{ps}$ of THz transients without (solid line)/with (dotted line) FSS structure

Lower inset: Fast Fourier transfoms of $\mathrm{THz}$ transients shown in upper inset

Experimental data: In Fig. 2, the detected THz pulse transient without the FSS structure in the setup is shown up to $330 \mathrm{ps}$ time delay. The data acquisition time was one minute. The upper inset of Fig. 2 shows the zoom into the first $10 \mathrm{ps}$ of two $\mathrm{THz}$ transients. One transient was recorded without, the other with, transmission through the FSS structure in the focused region of the THz setup (see Fig. 2, solid line/dotted line). The observed $\mathrm{THz}$ pulse duration is approximately $600-700 \mathrm{fs}$ in both cases. Comparing the transients, the FSS influence can be observed as amplitude attenuation and phase shift. The lower inset of Fig. 2 shows the corresponding fast Fourier transforms (FFTs) of the transients. In the dotted FFT curve an absorption line at $0.8 \mathrm{THz}$ can be observed, which is in agreement with the previously measured resonance of the FSS structure. In both FFT curves a water absorption line at 
1.2 THz is resolved and demonstrates that the usable spectral coverage extends to somewhat above this value. The $\mathrm{THz}$ emitter supports a spectral coverage of $\simeq 7 \mathrm{THz}$. Present limitations are the pulse duration of the SDL (370 fs here; sub-200 fs pulses were demonstrated in [4]) as well as its timing jitter resulting from a mechanical design very sensitive to acoustic perturbations. The SNR is currently at 56 , mainly due to the low available probe power for electro-optic detection. Efforts to increase the output power of the SDL using a tapered diode amplifier are under way [4] which will also enable use of modelocked SDLs for both pumping and probing. Longer averaging times, which can increase SNR, are currently prevented by a slow, thermally caused drift of the SDL repetition rate. A repetition rate of $\simeq 3 \mathrm{GHz}$ also for the pump laser will improve SNR, too; at present we use only every third probe pulse.

Conclusion: We present a THz-TDS system based on high-speed ASOPS using a femtosecond semiconductor disk laser and a modelocked Ti:sapphire laser as probe and pump laser, respectively. The system has a scan rate of $10 \mathrm{kHz}$ and offers more than $1.2 \mathrm{THz}$ spectral coverage at a SNR of 56. Significantly improved performance can be expected by straightforward mechanical optimisation of the SDL. Increasing the output power of femtosecond SDLs, e.g. by a tapered diode amplifier, will enable their use for both pumping and probing. We conclude that a rapid scanning, compact and low-cost THz-TDS system is feasible if modelocked SDL technology is combined with high-speed ASOPS. This may lead the way to higher-volume applications of THz-TDS outside of scientific laboratories.

(C) The Institution of Engineering and Technology 2010

30 October 2009

doi: $10.1049 / \mathrm{el} .2010 .3062$

R. Gebs, G. Klatt, T. Dekorsy and A. Bartels (Department of Physics and Center for Applied Photonics, University of Konstanz, Konstanz D-78457, Germany)

E-mail: Raphael.Gebs@uni-konstanz.de

P. Klopp and U. Griebner (Max Born Institute, Max-Born-Strasse 2A, Berlin D-12489, Germany)

\section{References}

1 Tonouchi, M.: 'Cutting-edge terahertz technology', Nature Photonics, 2007, 1, (2), pp. 97-105

2 Nagel, M., Richter, F., Haring-Bolivar, P., and Kurz, H.: 'A functionalized THz sensor for marker-frec DNA analysis', Phys. Med. Biol, 2003, 48, (22), pp. 3625-3636

3 Van Exter, M., Fattinger, Ch., and Grischkowsky, D.: 'Terahertz timedomain spectroscopy of water vapor', Opt. Lett., 1989, 14, (20), pp. $1128-1130$

4 Klopp, P., Griebner, U., Zorn, M., Klchr, A., Licro, A., Wcycrs, M., and Erbert, G.: 'Mode-locked InGaAs-AlGaAs disk laser generating sub200 -fs pulses, pulse picking and amplification by a tapered diode amplifier', Opt. Express, 2009, 17, (13), pp. 10820-10834

5 Klopp, P., Saas, F., Zorn, M., Weyers, M., and Gricbner, U.: '290-fs pulses from a semiconductor disk laser', Opt. Express, 2008, 16, (8), pp. $5770-5775$

6 Mihoubi, Z., Wilcox, K.G., Elsmere, S., Quarterman, A., Rungsawang, R., Farrer, I., Beere, H.E., Ritchie, D.A., Tropper, A., and Apostolopoulos, V.: 'All-semiconductor room-temperature terahertz time domain spectrometer', Opt. Lett., 2008, 33, (18), pp. 2125-2127

7 Bartels, A., Cerna, R., Kistner, C., Thoma, A., Hudert, F., Janke, C., and Dekorsy, T.: 'Ultrafast time-domain spectroscopy based on high-specd asynchronous optical sampling', Rev. Sci. Instrum., 2007, 78, (3), p. 035107

8 Klatt, G., Gebs, R., Janke, C., Dckorsy, T., and Bartcls, A.: 'Rapid scanning terahertz precision spectrometcr with more than $6 \mathrm{THz}$ spectral coverage', Opt. Express, 2009, 17, (27), to be published

9 Dreyhaupt, A., Winnerl, S., Dekorsy, T., and Helm, M.: 'High-intensity tcrahertz radiation from a microstructured large-area photoconductor', Appl. Phys. Lett., 2005, 86, (12), p. 121114

10 Zom, M., Klopp, P., Saas, F., Ginolas, A., Krüger, O., Griebncr, U., and Weyers, M.: 'Semiconductor components for femtosecond semiconductor disk lasers grown by MOVPE', J. Cryst. Growth, 2008, 310, (12), pp. 5187-5190

11 Fedotov, V.A., Rose, V., Prosvirnin, S.L., Papasimakis, N., and Zheludev, N.I.: 'Sharp trapped-mode resonances in planar metamatcrials with a broken structural symmetry', Phys. Rev. Lett., 2007, 99, (14), p. 147401

12 Klatt, G., Nagcl, M., Dekorsy, T., and Bartels, A.: 'Rapid and precise read-out of terahertz scnsor by high-speed asynchronous optical sampling', Electron. Lett., 2009, 45, (6), pp. 310-311 